\title{
Lack of correlation between tubular dentine cement penetration, adhesiveness and leakage in roots filled with gutta percha and an endodontic cement based on epoxy amine resin
}

\author{
Ricardo MACHADO ${ }^{1}$, Ulisses Xavier da SILVA NETO'1, Everdan CARNEIRO'1, Luiz Fernando FARINIUK¹, Vânia Portela \\ Ditzel WESTPHALEN ${ }^{1}$, Rodrigo Sanches CUNHA ${ }^{2}$
}

1- Department of Endodontics, Pontifical Catholic University of Paraná, Curitiba, PR, Brazil.
2- School of Dentistry, Restorative Dentistry, University of Manitoba, Winnipeg, Canada.

Corresponding address: Ricardo Machado - Rua Anibal Gaya, 898 - Casa 14 - Condomínio Nova Era - Centro -Navegantes - SC - Brazil - $88.375-000$ Phone/Fax: (55 47) 3319-1625 - Cel: (55 47) 8409-1561 - e-mail: ricardo.machado.endo@gmail.com

Submitted: March 15, 2013 - Modification: October 10, 2013 - Accepted: October 28, 2013

\section{ABSTRACT}

\begin{abstract}
bjective: To analyze possible correlations among tubular dentine cement penetration, adhesiveness and apical leakage in fillings performed with gutta percha and an endodontic cement based on epoxy amine resin. Material and Methods: Sixty similar, extracted human mandibular central incisors were irrigated, instrumented and filled following the same protocol. First, apical leakage was quantified by fluid filtration tests. Then, these same specimens were sectioned for analysis of tubular dentine cement penetration and the middle thirds were submitted to push-out tests to analyze the adhesiveness of the fillings. Results: In brief, the means and standard deviations with a confidence interval of 95\% were as follows: tubular dentine cement penetration (8.875 44.540$)$, adhesiveness $(4.441 \pm 2.683)$ and apical leakage $(0.318 \pm 0.215)$. The data were confronted using the Pearson's test $(P>0.05)$, and it was possible to prove that there was no correlation between (1) tubular dentine cement penetration and apical leakage ( $\left.r^{2}: 0.08276\right)$, (2) tubular dentine cement penetration and adhesiveness ( $\left.r^{2}:-0.2412\right)$ and (3) adhesiveness and apical leakage ( $\left.r^{2}: 0.1340\right)$. Conclusion: After analysis of these data, it could be observed that there exists no correlation among the variables analyzed in this study.
\end{abstract}

Keywords: Dentin. Adhesiveness. Leakage.

\section{INTRODUCTION}

In recent years, the evolution of Endodontics has broken several paradigms, driven by technological and techniques advances in all its phases of execution. However, despite all these technical and scientific developments, some concepts have not changed. The main objectives of root canal treatment continue to be the elimination of or reduction in the number of microorganisms within the root canal space, and the prevention of possible infection or reinfection ${ }^{18,28}$.

With this in mind, there is a clear interest in improving the effectiveness of the root canal filling techniques. Proof of this can be found in the great number of different systems recently developed to limit microbiological action capable of inducing or even causing resistance of a periapical lesion ${ }^{7,25}$.

The fillings failures observed in several studies, resulting from many different methodologies, have given rise to a global trend, evidenced by the literature, toward enhancing the ability of endodontic filling materials to project themselves into the dentinal tubules $2,7,31$. These materials not only act as antibacterial agents, theoretically speaking, but can also optimize the quality of the seals provided by these penetrations. This hypothesis is based on the concept that tubular dentine cement penetration considerably increases the contact surface of the filling material with the root canal walls, thereby improving the seal6,19,24. The possibility of improving the adhesiveness of 
the filling materials also has the ultimate goal of preventing leakage ${ }^{13}$.

However, these hypotheses have not yet been completely clarified in the literature, and few studies have been performed to investigate these possible correlations $4,6,12,13,19,23,24$.

Based on the above, this study aimed to analyze possible correlations between tubular dentine cement penetration, adhesiveness and leakage in roots filled with gutta percha and an endodontic cement based on epoxy amine resin.

\section{MATERIAL AND METHODS}

\section{Specimen selection}

After approval of the Ethics Committee (process 5314), sixty mandibular central incisor teeth with single straight canals, complete rhizogenesis, with no resorption or previous endodontic treatment, and free foraminal access, were provided by the university tooth bank and selected for this study. All this information was verified by clinical analysis and vestibulo-lingual and mesiodistal radiographs. The crowns were then removed using a low-speed steel cutting disc (Isomet-Buehler, Lake Bluff, IL, USA), standardizing all roots at $13 \mathrm{~mm}$ in length.

\section{Specimen preparation}

The accesses were performed using a taperedtip bur 3082 (KG Sorensen, Barueri, SP, Brazil). Working length was established by subtracting 1 $\mathrm{mm}$ from the point where the file was visible at the apical foramen. The coronal and middle thirds of each canal were prepared using Gates Glidden drills (Dentsply-Maillefer, Ballaigues, Switzerland) sizes 4,3 and 2 , by placing each instrument $2 \mathrm{~mm}$ deeper than the previous one. The apical foramina were standardized using real length instrumentation of the teeth up to instrument $25 \mathrm{~K}$-Flexofile (DentsplyMaillefer, Ballaigues, Switzerland), and the apical thirds were prepared with the Profile 04 System (Dentsply-Maillefer, Ballaigues, Switzerland) up to size 35 at working length. The canals were irrigated between each instrumentation with $2 \mathrm{~mL}$ of freshly prepared $2.5 \% \mathrm{NaOCl}$ plus a flush of $3 \mathrm{~mL}$ of $17 \%$ EDTA for $3 \mathrm{~min}$. Five milliliters of sterile water was used as a final rinse.

\section{Canal filling}

The prepared canals were filled using the lateral compaction technique. After drying with paper points, a size 20 file was used to place $10 \mu \mathrm{L}$ of an endodontic cement based on epoxy amine resin AH Plus (Dentsply-DeTrey, Konstanz, Germany) into the canal, using a counterclockwise rotation. The sealer was labeled with a $0.1 \%$ Rhodamine B dye (Sigma-Aldrich, St. Louis, MO, USA) for the purpose of further analysis by optical light microscopy.

A prefitted size 35, 0.04-taper gutta-percha cone (Dentsply-Maillefer, Ballaigues, Switzerland) was used as the master cone, and two accessory cones were used in addition. The filled roots were stored at $37^{\circ} \mathrm{C}$ and $100 \%$ humidity for 7 days, to allow the sealer to set.

\section{Apical leakage analysis by fluid filtration tests}

The fluid filtration method was used to determine apical leakage. The root apex was connected to a Luer-type metal needle by means of a plastic tube. The allowed leakage margin for the tested groups was quantified according to the movement of a small air bubble inside a $25 \mu \mathrm{L}$ micropipette (Microcaps-Fisher Scientific, Philadelphia, PA, USA). The inside of the pipette and the entire system was filled with distilled water and a pressure of 10 psi was applied. After making sure there was no leakage in the connections, the system was activated and balanced for 4 minutes. The volume of fluid was calculated by observing the air bubble displacements, expressed in $\mu \mathrm{L} / \mathrm{min}^{-1} .10 \mathrm{psi}$. Measurements were made at 2-minute intervals in a period of 8 minutes ${ }^{22,27}$.

\section{Tubular dentine cement penetration analysis by optical microscopy}

Each specimen was sectioned horizontally at 3, 6 and $8 \mathrm{~mm}$ from the apex, using a low-speed steel cutting disc (Isomet-Buehler, Lake Bluff, IL, USA). Three slices per root were created, resulting in a total of 180 slices. A standard polishing procedure using SiC paper $(200,300,400,600)$ followed by 3 $\mu \mathrm{m}$ diamond paste was employed on the coronally facing surface of each slice to produce a highreflection surface (Figures $1 A$ and $B$ ), and each slice was observed in a high-resolution stereomicroscope to acquire images at $1048 \times 1048$ pixels, covering the entire root surface. For each image, the outer perimeter of the root and the inner perimeter of the root canal walls were outlined and measured using the AxioVision Software 4.11 (Carl Zeiss, Jena, Germany) (Figures $1 \mathrm{C}$ and D). The total crosssectional area of the canal wall was obtained for each section by subtracting the value of the outer perimeter from the inner perimeter. The absolute cement penetration values (Figure 2) for each section were then converted into a percentage of cement penetration into dentinal tubules, by calculating the total cross-sectional area of the canal wall previously obtained in the high-resolution stereomicroscope. The percentages were averaged for each specimen. 
Adhesiveness analysis by mechanical push-out tests

Firstly, the thickness of each slice of the 60 sections corresponding to the middle third was measured with a digital caliper (Mitutoyo IP67Mitutoyo, Neuss, Germany). Then, the specimens were submitted individually to push-out bond strength tests using a universal-testing machine (EMIC DL200MF, São José dos Pinhais, PR, Brazil) at a speed of $0.5 \mathrm{~mm} / \mathrm{min}$ up to bond failure using a $0.50-\mathrm{mm}$ diameter stainless steel cylindrical plunger. The plunger tip was sized and positioned so that it contacted only the filling material. Because of the convergence of the root canal sections, the push-out force was applied from apical to coronal. The bond strength expressed in MPa at failure was calculated by dividing the load in newtons by the area of the bonded interface. The area of the bonded interface was calculated according to the following formula: area $=2 \pi r \times h$, where $\pi$ is kept constant at 3.14, and $\mathrm{r}$ and $\mathrm{h}$ are the radius and height measured in millimeters of the filling material

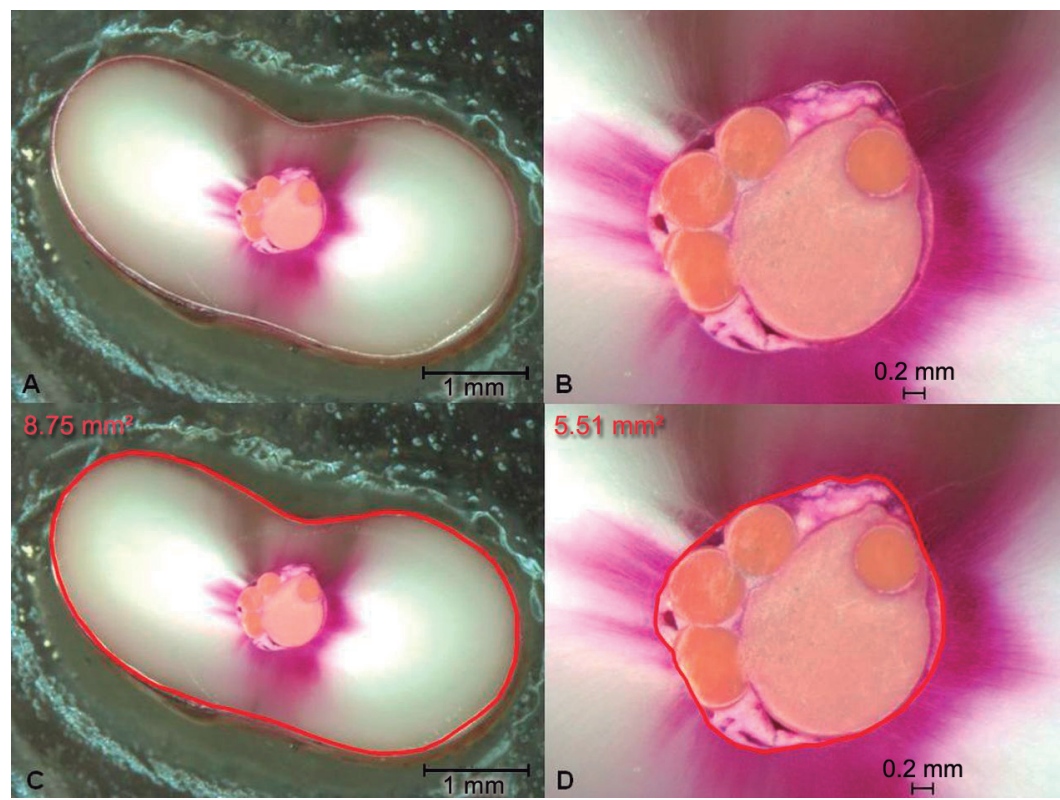

Figure 1- Coronal surfaces of the same slice after metallographic treatment. Total cross-sectional area (A) and canal cross-sectional area (B). The outer (C) and the inner (D) perimeter of the root canal walls outlined and measured using AxioVision Software 4.11 (Carl Zeiss, Jena, Germany)

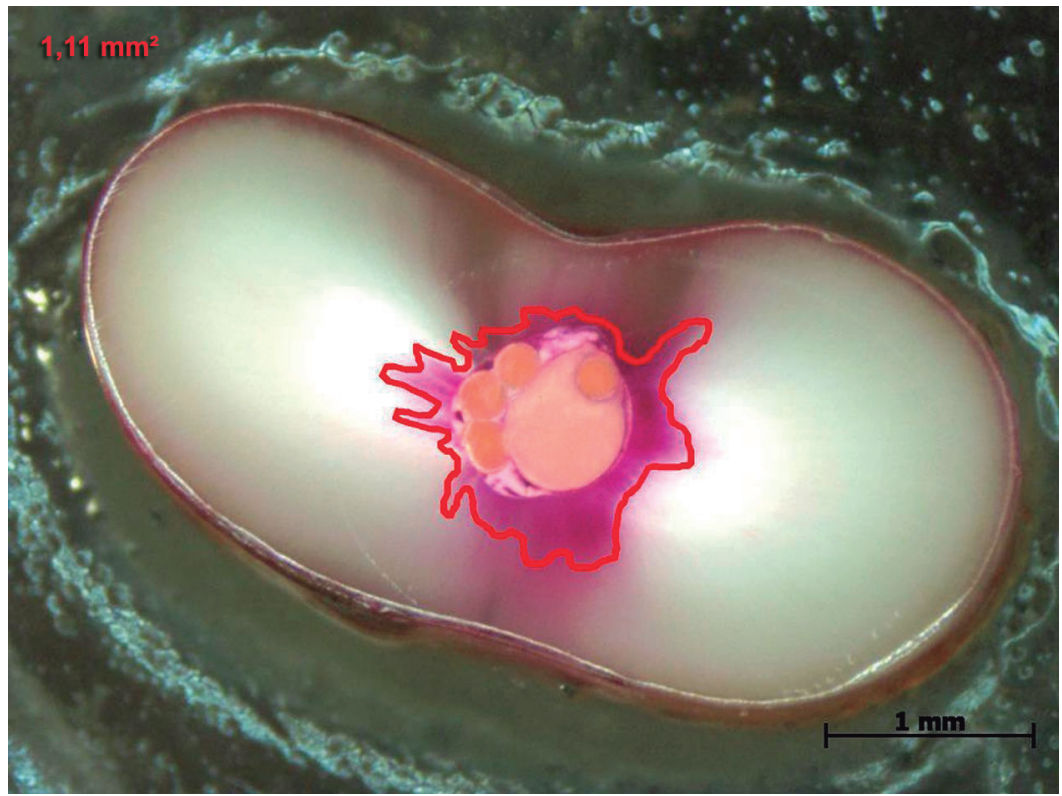

Figure 2- Absolute cement penetration values outlined and measured using AxioVision Software 4.11 (Carl Zeiss, Jena, Germany) 
that was pushed out ${ }^{1,23}$.

\section{Statistical analysis}

The results obtained were submitted to the Pearson test with a significance level of $95 \%$ $(p<0.05)$ to perform the correlation analysis among the variables of the study using the statistical software SPSS 11.5 (SPSS Inc., Chicago, IL, USA).

\section{RESULTS}

In isolated analyses of the three variables, the means and standard deviations, with a confidence interval of $95 \%$, were the following: tubular dentine cement penetration ( $8.875 \pm 4.540)$, adhesiveness $(4.441 \pm 2.683)$ and apical leakage (0.318 \pm 0.215$)$. The results obtained in the correlation analysis among these study variables were confronted and are shown in Figures 3, 4 and 5, respectively. There was no statistically significant correlation

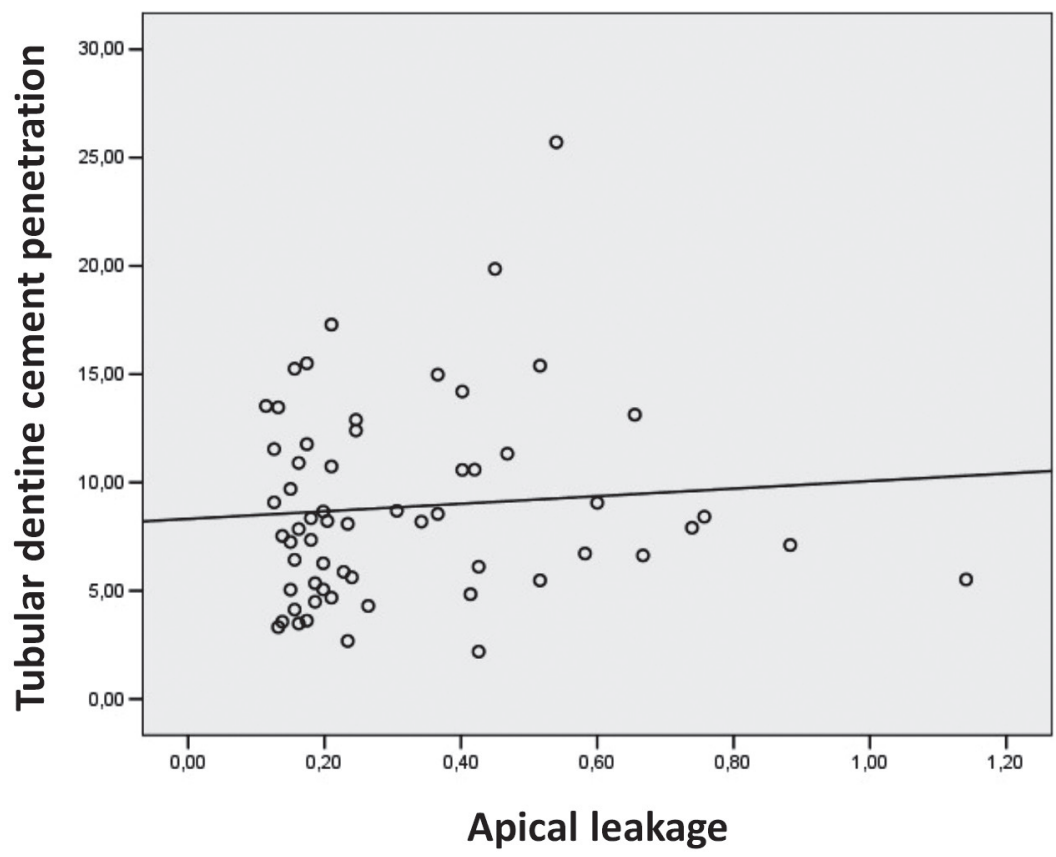

Figure 3- Representative image of the correlation analysis between tubular dentine cement penetration and leakage

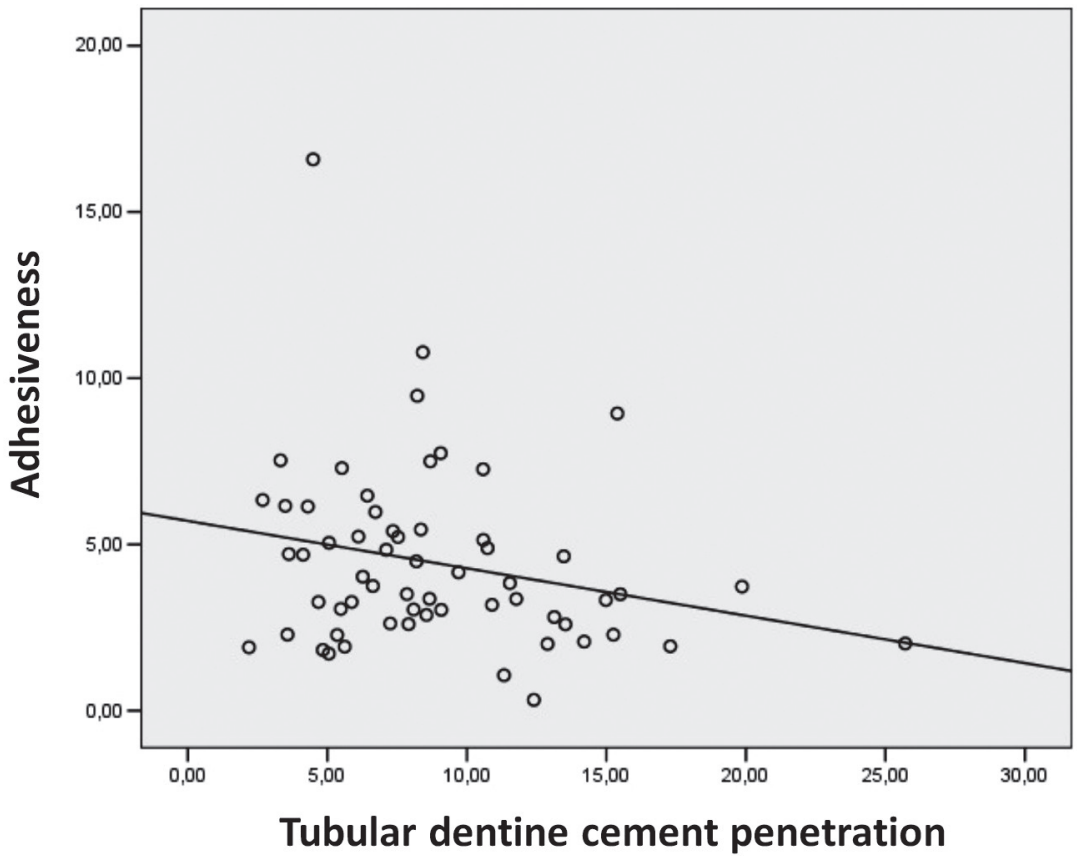

Figure 4- Representative image of the correlation analysis between adhesiveness and tubular dentine cement penetration 


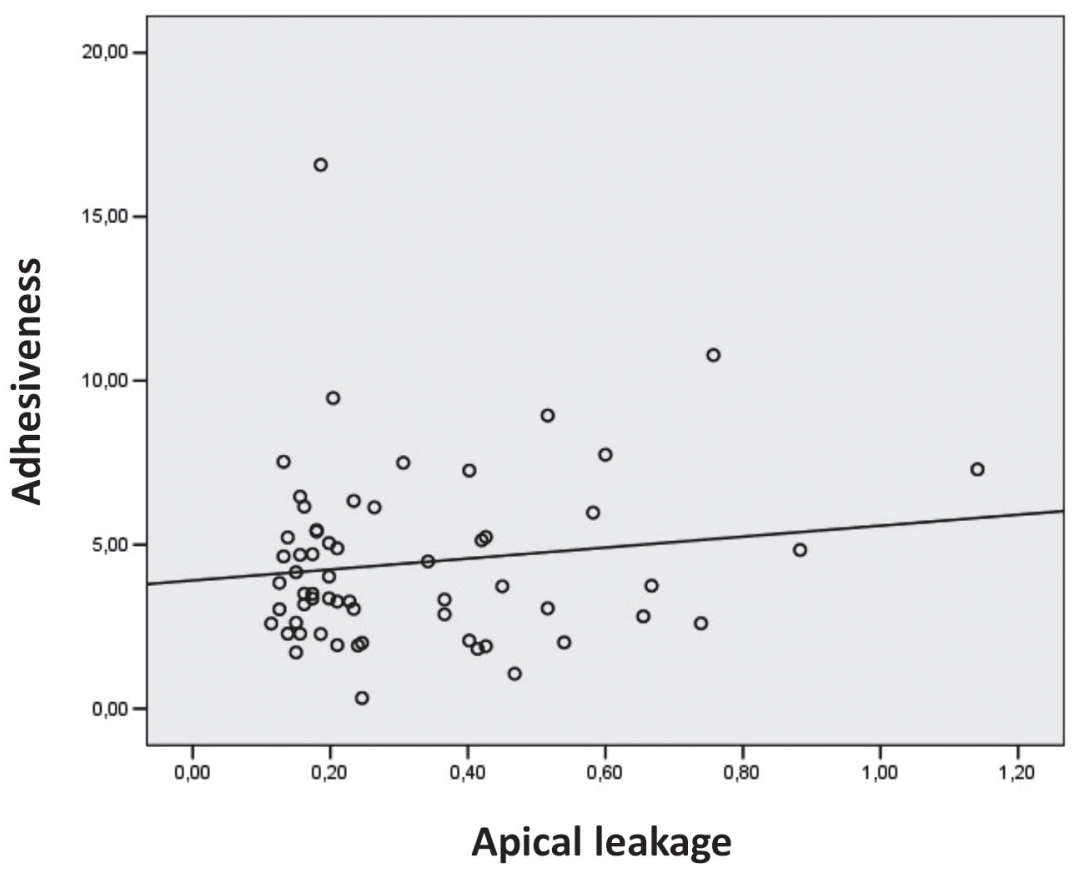

Figure 5- Representative image of the correlation analysis between adhesiveness and leakage

between tubular dentine cement penetration and apical leakage $\left(r^{2}: 0.08276\right)$, adhesiveness and tubular dentine cement penetration $\left(r^{2}:-0.2412\right)$ and adhesiveness and apical leakage $\left(r^{2}: 0.1340\right)$.

\section{DISCUSSION}

The hypothesis that tubular dentine cement penetration could improve sealability was firstly proposed in $1984^{29}$. Later, other studies were performed to address this premise ${ }^{3,9,16,20}$. These other studies were based on the original hypothesis that a better cement penetration into the dentinal tubules would somehow improve the quality of endodontic fillings. Therefore, previous reports that tubular dentine cement penetration was one of the most important factors to be considered in choosing the filling material justify the importance of conducting comparative studies on this matter ${ }^{10}$.

Most of the papers have analyzed the three variables studied in our paper - tubular dentine cement penetration ${ }^{2,21}$, adhesiveness ${ }^{5,8}$, and leakage ${ }^{14,15,22}$, merely comparing different materials. Only a few articles have tried to analyze possible correlations among these variables $4,6,12,13,19,23,24$.

Comparing four endodontic cements (Diaket, Endomethasone, CRCS and Ketac Endo) in relation to their projections into the dentinal tubules and their sealability using the same filling technique, Sen, et al. ${ }^{19}$ (1996) showed that the best results were obtained by different materials in isolated analyses. As in our results, it was not possible to observe any correlation between these two variables. The methods used (scanning electron microscopy and dye leakage test) were different from those adopted in this study (optical microscopy and fluid filtration test), but the main methodological point was to use the same specimens to analyze different points, an approach considered essential for analyzing possible correlations among two or more variables ${ }^{4}$.

Using a different protocol, another study investigated the dentine permeability obtained by two substances used in the final irrigation of root canals, namely, sodium hypochlorite and ethanol $95 \%$. This permeability was measured by analyzing the cement penetration using optical microscopy. In addition, specimens from the different study groups were also submitted to the fluid transport test to analyze the sealability of their fillings. Although a greater cement penetration and a lower level of leakage were observed in the group that used ethyl alcohol, this correlation was not verified statistically ${ }^{24}$, thereby showing results similar to those of our study ( $\left.r^{2}: 0.08276\right)$.

After analyzing our results and most of the studies performed to date, it appears that no correlation exists between tubular dentine cement penetration and sealability, or else the research methods used were unable to detect any correlation. The majority of the leakage tests, including fluid filtration, showed leakage only when there was at least one void extending from the apical to the coronal thirds. A root canal filling that looks poorly condensed on the radiograph may contain many "cul-de-sac"-type voids and no leakage. On the other hand, very small "through-and-through"type voids that are invisible on radiographs may be detected by the fluid filtration test and show considerable leakage rates ${ }^{26,30}$. In this case, the 
leakage rates would probably not be smaller if a major amount of sealer penetrated into the dentinal tubules. It seems that a more plausible hypothesis would be that this variable may be improved when there is a better adaptation of the cement to the canal walls.

Addressing adhesiveness and cement penetration, a classic study correlating these variables was performed to compare the adhesiveness of two filling systems (Gutta Percha/Kerr Pulp Canal Sealer EWT and Resilon/Epiphany) and the authors found more favorable results for the Resilon/Epiphany system. However, an analysis of images by SEM demonstrated no large cement penetrations into the dentinal tubules ${ }^{23}$. In view of this finding, the authors suggested that there was no effective correlation between cement penetration and adhesiveness, a conclusion corroborated by the results of this study. Our correlation results ( $r^{2}$ : -0.2412) and those mentioned above suggest that better adhesiveness is not related to a possible mechanical overlap provided by cement penetration or adhesive tags into the dentinal tubules. It seems legitimate to state that the establishment of a consistent hybrid layer in intratubular dentine plays a more important role in achieving better adhesiveness to the root dentine ${ }^{11,17}$.

Nagas, et al. ${ }^{12}$ (2007) analyzed the adhesiveness and leakage in root fillings comparing different methods of photoactivation (quartz halogen light for 40 seconds, light-emitting diode for 20 seconds, and plasma ARC for 6 seconds). It is worth highlighting that there were some important methodological differences in relation to the current study. The authors used different specimens to perform the push-out and leakage tests, and also carried out different statistical tests to analyze each variable individually. In the results related to adhesion, there were significant statistical differences among the three groups compared. However, when the leakage was analyzed separately, the differences between the two groups with the best results were not significant, suggesting a lack of correlation between these variables. This conclusion was demonstrated by the correlation results of our study as well $\left(r^{2}\right.$ : $0.1340)$.

To date, in the only study that has shown a positive correlation between adhesiveness and leakage ${ }^{13}$, there was an important methodological difference from those used in this research. The authors did not use gutta-percha or any solid material associated to the cement, which was used alone. Considering that the fluid filtration test shows leakage only when there is at least one void extending from the apical to the coronal thirds, it is possible that the sealer alone may be able to improve the sealability observed by this methodology.
The main point of our study was designed to produce a large experimental group. This is an important experimental design feature, because standard correlation analysis posits that any random factor affects only one subject, and not others. This requirement is violated when two or more different experimental groups are created, as was the case in all the aforementioned studies. In fact, there was no rationale to justify the creation of two or three experimental groups when the main goal was restricted to verifying a potential causeand-effect correlation. Therefore, when attempting to verify a potential cause-and-effect correlation, a single sizeable group should be created. However, even when a single well-standardized group was used, assessed through updated and refined experimental models, a correlation could not be established among the variables ${ }^{4}$.

\section{CONCLUSION}

Based on the experimental conditions and the results observed in this in vitro study, it is possible to prove that there are no correlations among tubular dentine cement penetration, adhesiveness and leakage.

\section{ACKNOWLEDGEMENTS}

The authors deny any conflicts of interest related to this study.

\section{REFERENCES}

1- Carneiro SM, Sousa-Neto MD, Rached FA Jr, Miranda CE, Silva SR, Silva-Sousa YT. Push-out strength of root fillings with or without thermomechanical compaction. Int Endod J. 2012;45:8218.

2- Chandra SS, Shankar P, Indira R. Depth of penetration of four resin sealers into radicular dentinal tubules: a confocal microscopic study. J Endod. 2012;38:1412-6.

3- De-Deus GA, Gurgel-Filho ED, Maniglia-Ferreira C, CoutinhoFilho T. The influence of filling technique on depth of tubule penetration by root canal sealer: a study using light microscopy and digital image processing. Aust Endod J. 2004;30:23-8.

4- De-Deus G, Brandão MC, Leal F, Reis C, Souza EM, Luna AS, et al. Lack of correlation between sealer penetration into dentinal tubules and sealability in nonbonded root fillings. Int Endod J. 2012;45:642-51.

5- Ebert J, Leyer A, Günther O, Lohbauer U, Petschelt A, Frankenberger $R$, et al. Bond strength of adhesive cements to root canal dentin tested with a novel pull-out approach. J Endod. 2011;37:1558-61.

6- Ghoddusi J, Dibaji F, Marandi S. Correlation between sealer penetration and microleakage following the use of MTAD as a final irrigant. Aust Endod J. 2010;36:109-13.

7- Hammad M, Qualtrough A, Silikas N. Evaluation of root canal obturation: a three-dimensional in vitro study. J Endod. 2009;35:541-4.

8- Imai Y, Komabayashi T. Properties of a new injectable type of root canal filling resin with adhesiveness to dentin. J Endod. 2003;29:20-3. 
9- Malyk Y, Kaaden C, Hickel R, Ilie N. Analysis of resin tags formation in root canal dentine: a cross sectional study. Int Endod J. 2010;43:47-56.

10- Mamootil K, Messer HH. Penetration of dentinal tubules by endodontic sealer cements in extracted teeth and in vivo. Int Endod J. 2007;40:873-81.

11- Mastoras K, Vasiliadis L, Koulaouzidou E, Gogos C. Evaluation of push-out bond strength of two endodontic post systems. J Endod. 2012;38:510-4.

12- Nagas E, Cehreli ZC, Durmaz V, Vallittu PK, Lassila LV. Regional push-out bond strength and coronal microleakage of Resilon after different light-curing methods. J Endod. 2007;33:1464-8.

13- Neelakantan P, Subbarao C, Subbarao CV, De-Deus G, Zehnder $M$. The impact of root dentine conditioning on sealing ability and push-out bond strength of an epoxy resin root canal sealer. Int Endod J. 2011;44:491-8.

14- Oddoni PG, Mello I, Coil JM, Antoniazzi JH. Coronal and apical leakage analysis of two different root canal obturation systems. Braz Oral Res. 2008;22:211-5.

15- Ozok AR, van der Sluis LW, Wu MK, Wesselink PR. Sealing ability of a new polydimethylsiloxane-based root canal filling material. J Endod. 2008;34:204-7.

16- Patel DV, Sherriff M, Ford TR, Watson TF, Mannocci F. The penetration of RealSeal primer and Tubliseal into root canal dentinal tubules: a confocal microscopic study. Int Endod J. 2007;40:67-71.

17- Prado M, Simão RA, Gomes BP. Effect of different irrigation protocols on resin sealer bond strength to dentin. J Endod. 2013;39:689-92.

18- Ricucci D, Loghin S, Siqueira JF Jr. Exuberant Biofilm infection in a lateral canal as the cause of short-term endodontic treatment failure: report of a case. J Endod. 2013;39:712-8.

19- Sen BH, Pişkin B, Baran N. The effect of tubular penetration of root canal sealers on dye microleakage. Int Endod J. 1996;29:238.

20- Sevimay S, Dalat D. Evaluation of penetration and adaptation of three different sealers: a SEM study. J Oral Rehabil. 2003;30:951-5.

21- Shokouhinejad N, Sabeti M, Gorjestani H, Saghiri MA, Lotfi $M$, Hoseini A. Penetration of Epiphany, Epiphany self-etch and $A H$ Plus into dentinal tubules: a scanning electron microscopy study. J Endod. 2011;37:1316-9.
22- Silva Neto UX, Moraes IG, Westphalen VP, Menezes R, Carneiro $E$, Fariniuk LF. Leakage of 4 resin-based root-canal sealers used with a single-cone technique. Oral Surg Oral Med Oral Pathol Oral Radiol Endod. 2007;104:53-7.

23- Skidmore LJ, Berzins DW, Bahcall JK. An in vitro comparison of the intraradicular dentin bond strength of Resilon and guttapercha. J Endod. 2006;32:963-6.

24- Stevens RW, Strother JM, McClanahan SB. Leakage and sealer penetration in smear-free dentin after a final rinse with $95 \%$ ethanol. J Endod. 2006;32:785-8.

25- Tanomaru-Filho M, Sant'Anna A Jr, Berbert FL, Bosso R, Guerreiro-Tanomaru JM. Ability of gutta-percha and Resilon to fill simulated lateral canals by using the Obtura II system. J Endod. 2012;38:676-9.

26- Van der Sluis LW, Wu MK, Wesselink PR. An evaluation of the quality of root fillings in mandibular incisors and maxillary and mandibular canines using different methodologies. J Dent. 2005;33:683-8.

27- Vasiliadis L, Kodonas K, Economides N, Gogos C, Stavrianos C. Short and long-term sealing ability of Gutta-flow and $\mathrm{AH}$-Plus using an ex vivo fluid transport model. Int Endod J. 2010;43:377-81. 28- Vieira AR, Siqueira JF Jr, Ricucci D, Lopes WS. Dentinal tubule infection as the cause of recurrent disease and late endodontic treatment failure: a case report. J Endod. 2012;38:250-4.

29- White RR, Goldman M, Lin PS. The influence of the smeared layer upon dentinal tubule penetration by plastic filling materials. J Endod. 1984;10:558-62.

30- Wu MK, Bud MG, Wesselink PR. The quality of single cone and laterally compacted gutta-percha fillings in small and curved root canals as evidenced by bidirectional radiographs and fluid transport measurements. Oral Surg Oral Med Oral Pathol Oral Radiol Endod. 2009;108:946-51.

31- Zapata RO, Bramante CM, Bernardineli N, Graeff MS, Garcia RB, Moraes IG, et al. A preliminary study of the percentage of sealer penetration in roots obturated with the Thermafil and RealSeal-1 obturation techniques in mesial root canals of mandibular molars. Oral Surg Oral Med Oral Pathol Oral Radiol Endod. 2009;108:961-8. 